\title{
Inventer le marché par la conservation
}

Le cas des viandes de lait en Corse et en Sardaigne

\section{Nicolas Lacombe}

\section{(2) OpenEdition}

Journals

Édition électronique

URL : https://journals.openedition.org/tc/8816

DOI : $10.4000 /$ tc. 8816

ISSN : 1952-420X

Éditeur

Éditions de l'EHESS

\section{Édition imprimée}

Date de publication : 17 mai 2018

Pagination : 136-147

ISBN : 978-2-7132-2750-9

ISSN : 0248-6016

Référence électronique

Nicolas Lacombe, «Inventer le marché par la conservation », Techniques \& Culture [En ligne], 69 | 2018, mis en ligne le 17 mai 2020, consulté le 29 septembre 2022. URL : http://journals.openedition.org/tc/ 8816 ; DOI : https://doi.org/10.4000/tc.8816 


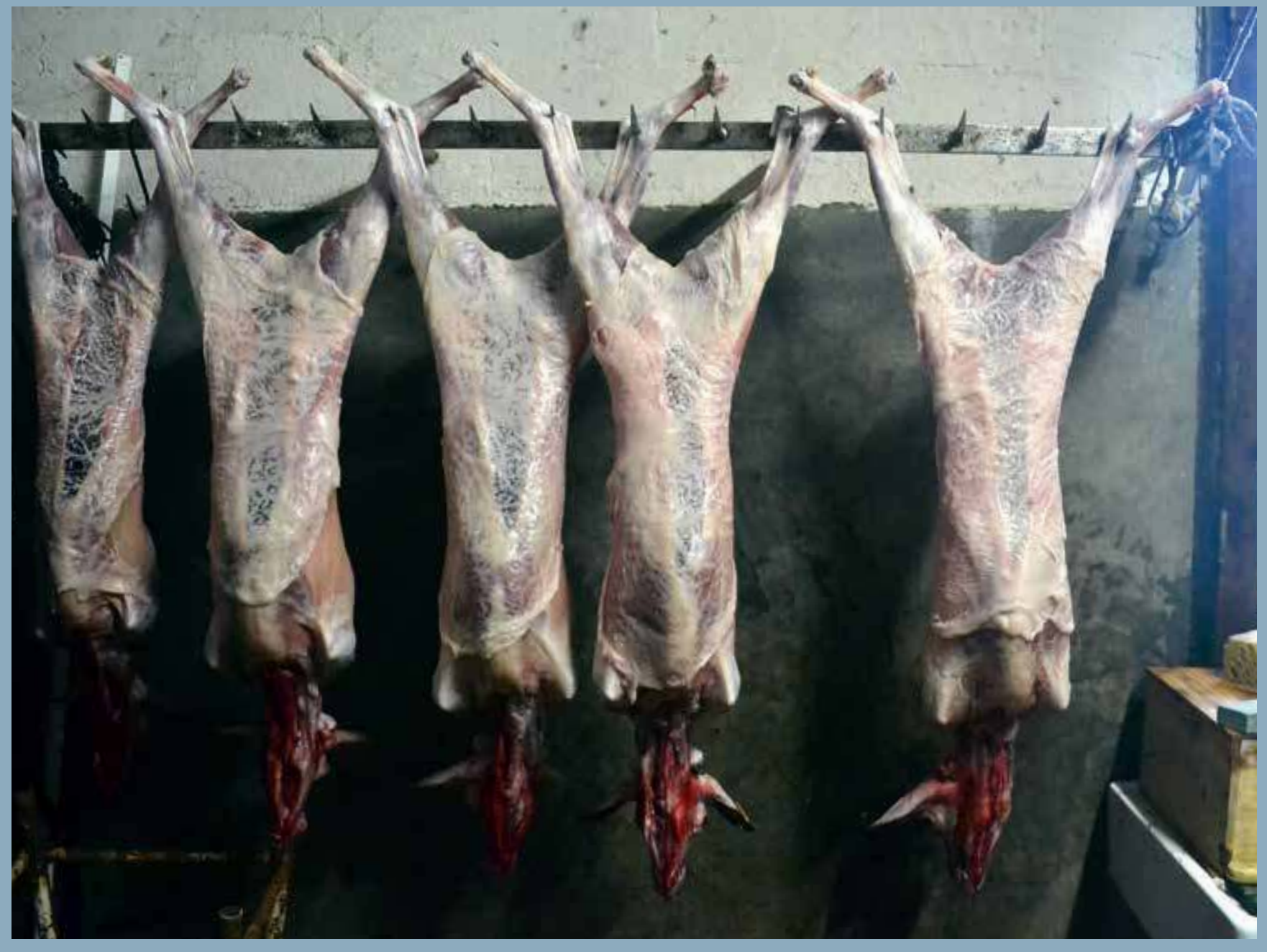




\section{Inventer le marché par la conservation}

\section{Le cas des viandes de lait en Corse et en Sardaigne}

L'inventaire du patrimoine culinaire de la France, lancé par le ministère de la Culture en 1989, répertorie dans son édition consacrée à la Corse une production assez confidentielle, pour ne pas dire méconnue: les agneaux et cabris de lait. Ils appartiennent à une catégorie de production bien particulière en tant que produits dérivés d'une activité principale. Les «viandes de lait» sont issues d'élevages dont la vocation principale est la production de fromages (Brocciu, Venachese); à ce titre, on les qualifie souvent de «sous-produits». Aujourd'hui pourtant, cette production caractéristique de l'élevage ovin et caprin prend une place essentielle au titre de la valorisation du patrimoine alimentaire corse et sarde. Les démarches engagées en vue de l'obtention de signes officiels d'identification de la qualité et de l'origine (SIQO) témoignent de relances visant à en faire de véritables « coproduits» (Delfosse 2010). Cette reconnaissance passe par de nouvelles formes de segmentation des marchés (tourisme, grande distribution). En effet, si la consommation familiale des viandes de lait à Noël et à Pâques relevait jusqu'à présent d'une pratique prétendument immuable, le choix actuel de promouvoir des «hamburgers nustrale ${ }^{1}$ » à base d'agneau témoigne d'une recomposition de l'espace social de l'aliment (Poulain 2002). Dans ces dynamiques destinées à faire revivre un produit par l'accès à de nouveaux marchés, la conservation constitue une médiation sociotechnique, qui reste peu étudiée, entre l'acte de produire et celui de vendre (Akrich 1993). En quoi et comment la conservation intervient-elle dans le processus de requalification marchande des viandes de lait? Quel est le rôle de l'emballage dans de telles opérations?

Cette recherche s'inscrit dans la continuité des travaux relatifs à la qualité agroalimentaire (Stanziani 2003), envisagée comme l'ensemble des caractéristiques données à un bien en vue de le faire circuler vers et au sein d'un espace marchand (Callon et al. 2000, Anzalone 2005), de l'animal vif à la carcasse (Escala 2006). Aujourd'hui, l'une des approches scientifiques se focalise sur l'avènement d'une économie des singularités (Karpik 2007), fondée sur la différenciation 
économique des productions agroalimentaires. En Corse et en Sardaigne, la mobilisation des $\mathrm{SIQO}^{2}$ fait appel à la question de l'origine et la qualité s'y définit habituellement par la spécification des conditions de production (race, alimentation, etc.) (Sylvander 1995). Le temps et le lieu font alors preuve d'authenticité, comme le soulignent les analyses en termes de «terroir» (Bérard \& Marchenay 2000). Mais cette approche nous semble écarter certaines dimensions relatives à la qualité agroalimentaire qui s'appuie aussi sur d'autres dispositifs de différenciation du marché des viandes de lait. En effet, si les critères mobilisés dans les relances de produits dits «traditionnels» reposent en partie sur des pratiques d'élevage (allaitement au lait maternel sous la mère), la capacité à faire revivre un produit oublié revient largement à le faire exister au sein d'une économie formelle en marge de laquelle il s'est tenu - les viandes de lait étant associées à une commercialisation en vente directe. L'accès au marché pose alors de façon centrale la question de la conservation de ces produits. Abattre, découper, emballer ou surgeler font référence à des pratiques qui nous invitent à renouveler l'analyse du travail de qualification (Cochoy \& Dubuisson-Quellier 2000). La conservation doit avant tout rendre possible un échange marchand en voie de recomposition (Callon 2013). Qu'elle soit envisagée en

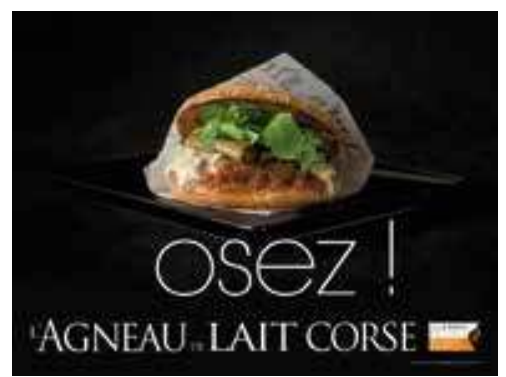
termes de saisonnalité lorsque le touriste en été s'éprend de produits locaux, en référence au commerce urbain qui déplace les lieux de vente, ou d'un point de vue réglementaire afin de répondre à des enjeux de sécurité alimentaire, la conservation permet d'inventer le marché.

À partir d'un travail d'enquête réalisé en Corse et en Sardaigne (Lacombe 2015), j'analyse la transition d'un modèle de qualification domestique du produit, construit en référence à des traditions alimentaires locales, vers un marché s'appuyant sur de nouvelles formes de transactions où l'acte alimentaire prend une tout autre dimension. J'y compare les situations des viandes de lait dans ces deux régions italiennes et françaises sur la base de 74 entretiens

L'agneau de lait façon «Hamburger»

Dans la perspective de relancer la filière, l'office de développement agricole et rural de la Corse a lancé en 2014 une campagne promotionnelle afin de reconquérir les consommateurs, en s'adressant ici à un public jeune. réalisés entre 2011 et 2015 auprès des différents acteurs de la filière (éleveurs, bouchers, abattoirs, coopératives, grande distribution, restaurateurs, consommateurs). La mise en perspective du cas des agneaux avec celui des cabris de lait permet de rendre compte de la différenciation de leur trajectoire marchande. Ces singularités sont l'occasion de positionner les techniques et objets de conservation dans le champ de la sociologie des conventions qui envisage la construction sociale de la qualité selon plusieurs régimes de production et d'échange (Diaz-Bone \& Thévenot 2010). Nous distinguerons les conventions domestiques qui concernent les caprins et les produits fermiers, basées sur la réputation individuelle activée lors de la vente directe, des conventions industrielles qui concernent les ovins vendus sous SIQO et qui se réfèrent à la standardisation et aux circuits longs (Sylvander 1995).

Dans un premier temps, il s'agit de contextualiser les usages traditionnels du produit et ses implications sur l'emploi des techniques de conservation (vente de carcasses entières en frais). Dans un second temps, je propose d'analyser l'émergence de nouvelles pratiques (découpe, emballage, surgélation) au regard de l'évolution des formes d'échange et de consommation auxquelles ces viandes de lait donnent lieu. 


\section{D'une " communauté alimentaire " vers la différenciation des identités de consommation}

Si les viandes de lait ont longtemps été associées à une commercialisation saisonnière à Noël et à Pâques, articulée aux traditions alimentaires et au fonctionnement des élevages, agneaux et cabris de lait connaissent aujourd'hui des trajectoires de commercialisation distinctes. La question de la conservation se pose alors en des termes différents selon la nature des marchés.

\section{Les viandes de lait : caractéristiques et pratiques alimentaires}

Les viandes de lait sont des productions issues d'élevages laitiers. Les éleveurs transforment le lait en fromages, mais produisent aussi des agneaux et cabris. La gestion des naissances se traduit par la composition de deux lots: d'une part la sélection des agnelles ou chevrettes de renouvellement qui vont entrer dans le troupeau productif laitier, et d'autre part les animaux destinés à la boucherie, qui nous intéressent ici. Les agneaux et cabris sont allaités au lait maternel sous la mère durant 30 à 45 jours, pour un poids carcasse moyen d'environ 4,5 à $7 \mathrm{~kg}$, ce qui les différencie des agneaux lourds ou des chevreaux issus de races à viande spécialisées (ex: l'agneau de Sisteron). Les cycles de production sont corrélés à la consommation locale des viandes de lait. On distingue les mises bas principales (novembre), des mises bas secondaires (février-mars), permettant ainsi de commercialiser les agneaux et cabris à Noël et à Pâques. Cette saisonnalité alimentaire tient à un héritage culturel et religieux plaçant la consommation de ces viandes durant la Nativité et la Semaine sainte. Ce rituel de consommation scelle à la fois la perpétuation d'une communauté dans des régions aux identités culturelles marquées, et donne aux familles l'occasion de se retrouver autour d'une tablée où différents plats élaborés à partir de l'ensemble des pièces de la carcasse rythment le repas. C'est un animal entier qui est acheté et qui convient très bien à une table de 5 à 8 personnes.

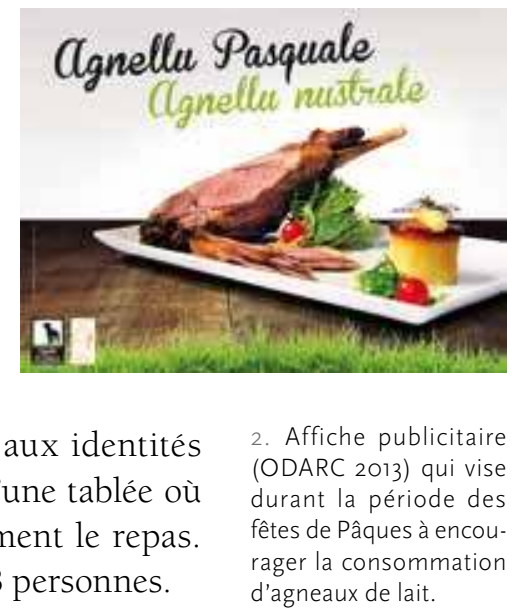

\section{La vente directe chez les producteurs fermiers}

La vente directe à la ferme a prédominé jusqu'au début des années 1980. Ce régime d'échange s'appuyait sur des relations de confiance interpersonnelle entre les éleveurs et une clientèle d'habitués. C'est un modèle qui reste encore très présent concernant la vente des cabris uniquement ${ }^{3}$ (Lacombe et al. 2012). L'abattage fermier est très répandu chez les chevriers. L'une des raisons qui explique cette pratique tient à un manque de professionnalisme dans les structures agréées, qui emploient un personnel peu qualifié et dont les équipements ne sont pas adaptés aux viandes de lait ${ }^{4}$. «Passer par les abattoirs c'est bien, tant que ce sont 
3. Décollage de la peau

Le décollage de la peau, ici pratiqué par un éleveur, est une phase essentielle du travail de la carcasse. les gestes employés permettent d'éviter un déchirement du gras de couverture qui joue un rôle important lors de la cuisson.

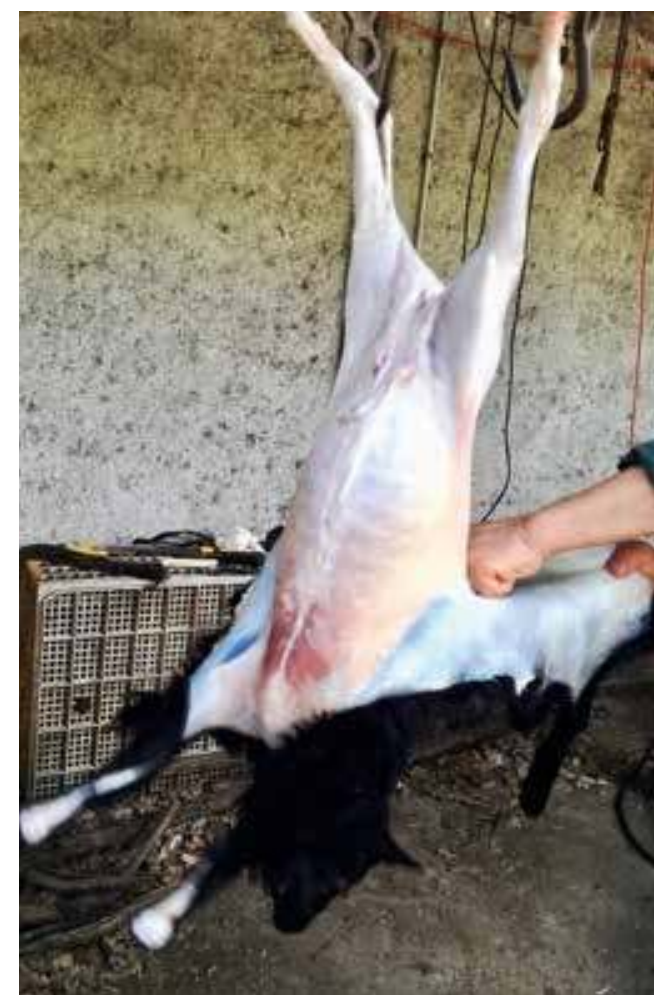

des professionnels, mais si je récupère un lot que je ne peux pas vendre, je préfère tuer chez moi » (éleveur, Corte).

L'éleveur abat lui-même ses animaux, chez lui, puis les vend directement en carcasses entières aux particuliers, après quoi ils seront consommés en frais lors des fêtes. Les éléments d'appréciation de la qualité des carcasses permettent aux éleveurs d'évaluer l'état de l'animal par un jugement visuel sur les tissus, la chair, la coloration, articulé à des gestes spécifiques. L'échange en carcasse entière suppose une réelle compétence de la part des consommateurs, pour le travail domestique de découpe et de préparation des viandes. Or cette aptitude se révèle fragile chez les plus jeunes qui tendent à préférer des produits de substitution plus simples à préparer (dinde, chapon). Une telle évolution en matière de consommation traduit une transition vers un tout autre modèle dans lequel les techniques de conservation renvoient au travail professionnel de découpe des carcasses.

\section{L'adoption de signes de qualité comme nouveau rapport au marché}

La professionnalisation du secteur ovin a fait entrer en scène de nouveaux opérateurs et infrastructures, soumettant les agneaux à un régime de qualification renégocié. Le groupement de Roquefort ${ }^{5} \mathrm{et}$ l'association régionale des éleveurs ovins (AREO) sont les deux principaux représentants de la filière en Corse et apparaissent comme de nouveaux prescripteurs de la qualité (Cochoy \& DubuissonQuellier 2000). Ils collectent et commercialisent les produits de leurs adhérents mais déterminent aussi de nouveaux choix de segmentation des marchés. Ces opérateurs sont soumis à des règlements sanitaires et doivent répondre à l'exigence de passer par des abattoirs agréés où les lots d'animaux sont soumis à des dispositifs de contrôle relatifs à la qualité des carcasses. Le régime de qualification domestique des produits est progressivement remplacé par une nouvelle logique de différenciation des marchés associée à la valorisation de la qualité liée à l'origine (Karpik 1989). Le signe de qualité obéit à une logique de spécification marchande du produit, c'est-à-dire qu'il s'adresse à de nouvelles catégories de consommateurs (Lambert 1986), n'entretenant pas de liens avec les traditions alimentaires locales. Tourisme, restauration hors domicile, grandes et moyennes surfaces, boucheries spécialisées sont des circuits de vente qui conduisent à repenser les techniques de conservation. 


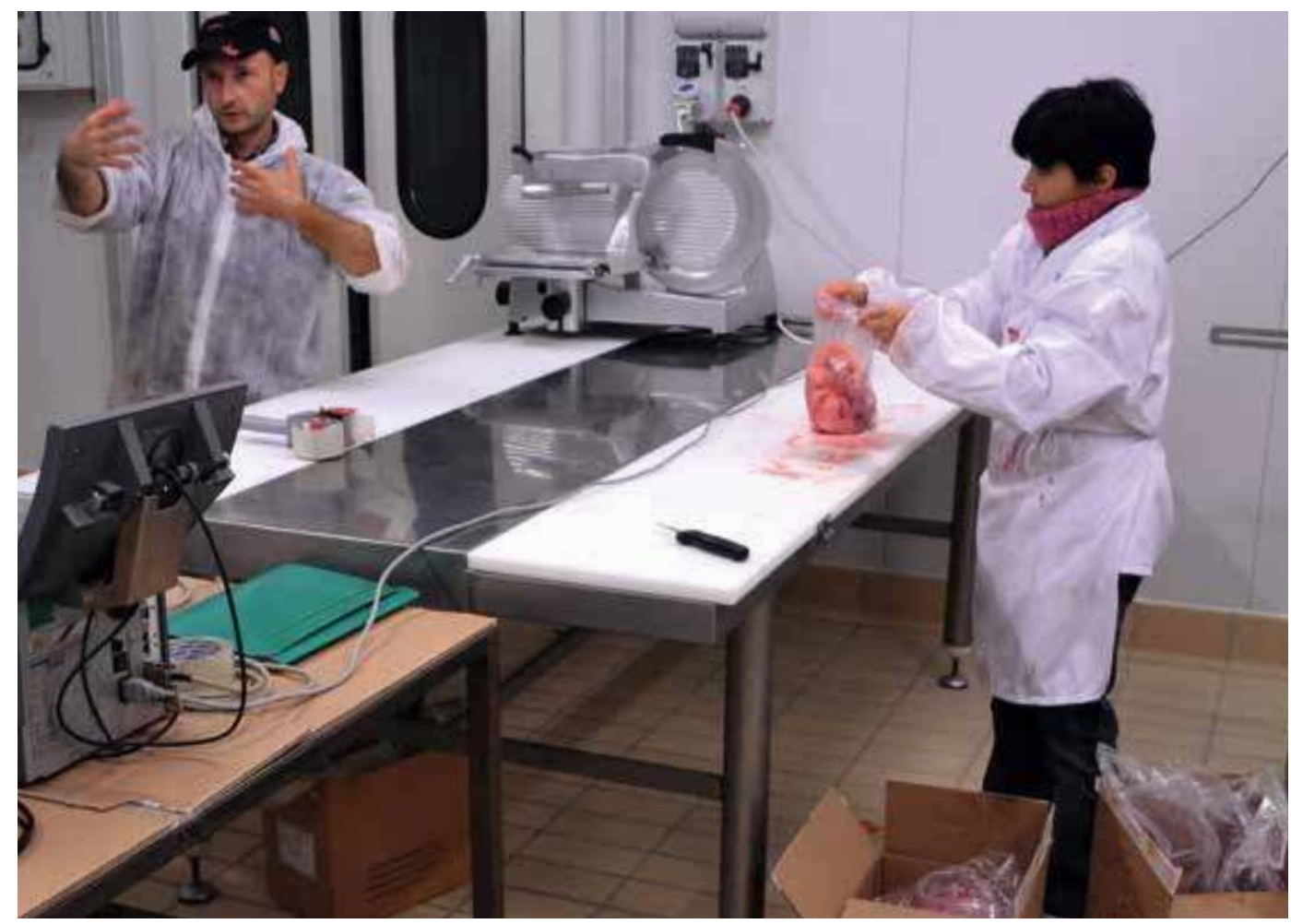

4. Emballage des agneaux sardes sous IGP

Depuis l'adoption de l'indication géographique en Sardaigne, le travail dans les abattoirs et ateliers de conditionnement est particulièrement rigoureux et vise à assurer la sécurité alimentaire des consommateurs sur des circuits longs.

\section{Surgeler, découper, contrôler : faire circuler le produit autrement}

Les acteurs mobilisés autour des viandes de lait, après s'être centrés durant une cinquantaine d'années vers la valorisation fromagère, s'investissent aujourd'hui dans le domaine de la qualification des viandes afin d'en faire un véritable coproduit plus qu'un sous-produit. Or les pratiques alimentaires ont profondément évolué tout autant que les normes qui régissent l'accès au produit, et les techniques de conservation sont ainsi appelées à jouer un nouveau rôle.

\section{Du frais au surgelé : reporter la consommation}

Les industries de Roquefort ont introduit en Corse le désaisonnement des troupeaux au début du siècle dernier, afin d'obtenir un lait de contre-saison pour compenser l'absence de production en automne et en hiver dans leur propre bassin de production (Delfosse $\&$ Prost 1998) ${ }^{6}$. La précocité actuelle des mises bas rend impossible une commercialisation des agneaux de lait à Noël et à Pâques, ce qui a induit la construction d'un débouché alternatif vers la Sardaigne, avec une 


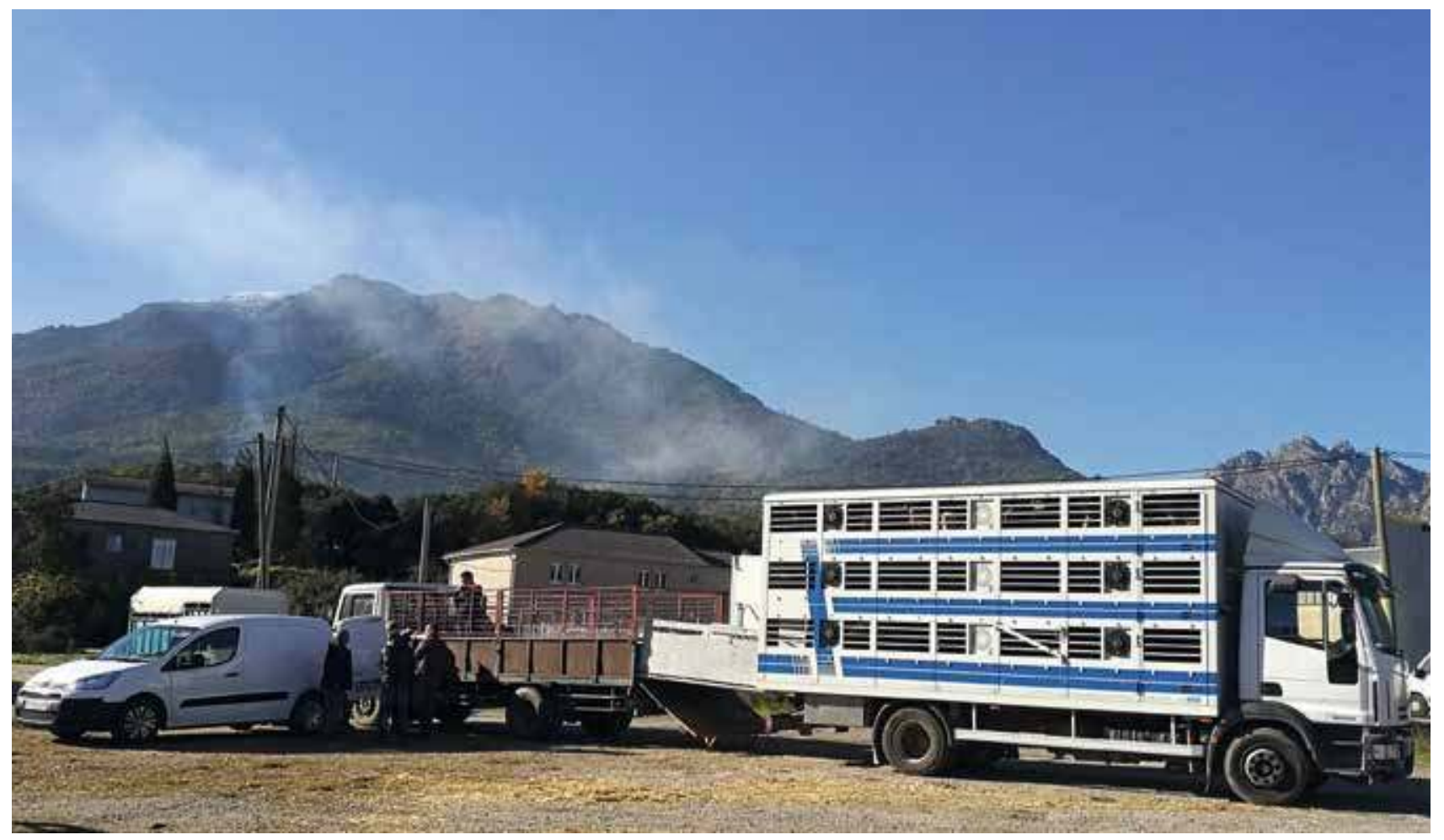

5. Acheminement des agneaux vers la Sardaigne

Les agneaux issus de mises bas décalées sont livrés en vif vers les abattoirs sardes et ce découplage géographique des fonctions a long. temps conduit les opérateurs corses à ne pas se poser la question de la conservation des carcasses. forte dépréciation économique du produit. Les opérateurs corses ont alors vu dans la surgélation l'opportunité de reporter la consommation de viandes de lait à destination du tourisme local, rompant ainsi avec la vente de viandes fraîches.

Laliment à attribut local fait ici sens auprès des populations touristiques durant la période estivale, où il est consommé dans les restaurants et fermes-auberges (Senil et al. 2014). La société d'entreposage et de dépôt de denrées alimentaires (SEDDA) réalise cette prestation de stockage pour le compte des coopératives. Le marché touristique est plus flexible dans la mesure où il est structuré par des consommateurs détachés des traditions alimentaires, permettant à ce produit de quitter les tables de fêtes. Pour les metteurs en marché, la concentration du marché à Noël et à Pâques est contraignante puisqu'elle ne permet pas d'avoir de souplesse au regard de la période des mises bas. "On peut jouer sur plusieurs segments de marché grâce à la surgélation, ça nous permet de diversifier nos stratégies selon l'échelonnement des naissances durant l'année» (AREO).

Le produit, jusqu'ici encastré dans une société locale, quitte par la voie de la surgélation un cercle d'initiés au profit d'une consommation de type «découverte». La conservation permet alors de résoudre la tension entre la saisonnalité de l'élevage et des marchés. 


\section{Découper et conditionner des portions: l'émergence de l'emballage}

La reconquête locale de la consommation se fait aussi au travers de l'accès à la grande distribution qui permet d'écouler des lots importants d'agneaux de lait. La fragilisation des savoirs domestiques de découpe des carcasses favorise la commercialisation de produits prédécoupés. Gigots, épaules, côtelettes représentent des pièces individuelles, préférées par les consommateurs pour leur facilité de préparation. Un piéçage se met dès lors en place afin de répondre à de nouvelles pratiques et calendriers de consommation: une consommation courante hors périodes festives. Indissociable de la découpe, le conditionnement devient une opération incontournable. Frais réfrigéré sous-vide, film d'emballage plastique, barquettes sont autant de techniques de conditionnement répondant à des logiques de consommation spécifiques, qui mettent au jour les contrastes entre pratiques alimentaires urbaines et rurales, comme le souligne Patrizia Pitzalis, la Directrice du Consortio per la tutela dell' IGP agnello sardo (CONTAS) : «Dans le milieu rural, cuisiner des bêtes entières ça se pratique encore, mais en ville, les profils de consommation sont différents, il faut portionner. »

Les techniques les plus abouties développées en Sardaigne sont liées à l'usage de l'emballage sous atmosphère modifiée, solution optimale pour étirer le temps de l'alimentation avec des dates limite de consommation étendues à 30 jours. Les plats pré-cuisinés et conditionnés sous vide se développent et offrent des solutions « micro-ondables » répondant au raccourcissement du temps de préparation des aliments, à une individualisation des modes de consommation et au développement de la restauration hors domicile. Loin de se réduire à une fonction informationnelle produisant un lien d'échange (Cochoy 2002), les emballages employés constituent également une réponse technique à la volonté de toucher des marchés alimentaires très différents.

\section{Abattre à la ferme, entre tension et compromis sanitaire négocié}

Conserver, c'est aussi fournir au consommateur un produit sain, exempt de tout risque sanitaire. Pour cela, la France s'est dotée d'une réglementation qui interdit la pratique de l'abattage fermier, encore il y a peu tolérée. Cela s'est traduit par une centralisation du contrôle par les Directions des Services Vétérinaires dans des infrastructures agréées. Or cette exigence va à l'encontre des producteurs fermiers qui, pour certains d'entre eux, souhaitent se doter d'infrastructures d'abattage adaptées. Les directives européennes prévoient en ce sens des mesures dites de flexibilité sanitaire dont fait application l'Italie et que l'on retrouve en Sardaigne. En France la confédération paysanne a entrepris de faire amender les règles nationales en s'inspirant de ce régime juridique. Celui-ci garantit une prise en charge du risque sanitaire à l'échelle des exploitations. Les financements alloués aux éleveurs leur ont permis de bénéficier d'équipements et de bâtiments adaptés (chambre froide, rail de circulation, système de nettoyage). Le régime sanitaire est basé sur le standard $\mathrm{HACCP}^{7}$, avec plusieurs points de contrôle: choix des matériaux (inox, carrelage), formations aux règles d'hygiène et de sécurité, évaluation pré et post-mortem des 
animaux par les vétérinaires. Ce compromis institutionnalisé permet aux éleveurs de valoriser une production fermière soit par de la vente directe auprès de particuliers, soit par le biais de structures d'agritourisme, et ce en dehors de la «logique terroir» propre aux signes de qualité (Delfosse \& Bernard 2007). «Faire le choix du signe de qualité, c'est implicitement adhérer au passage par des abattoirs agréés, ce qui serait contraire à notre profession de producteur fermier» (éleveur, Pietralba).

Dans cette perspective, on constate que les normes et représentations associées à la conservation ne sont pas stabilisées et font l'objet de tensions entre plusieurs régimes de qualification marchande.

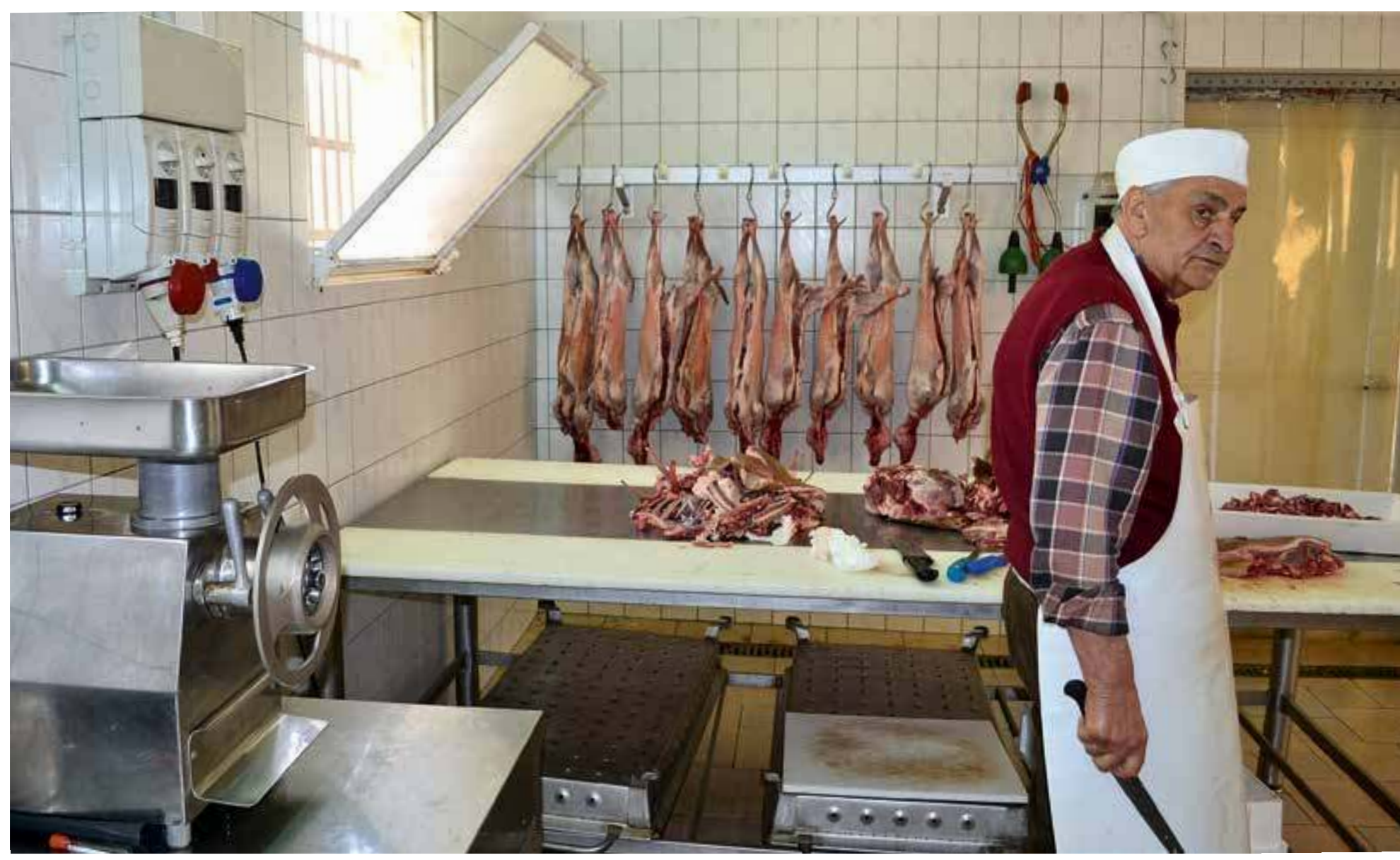


Derrière tout l'équipement technologique mobilisé (froid, date limite de consommation, emballage, etc.), la conservation apparaît comme un «objet intermédiaire», en tant qu'entité physique vectrice de liens entre producteurs et consommateurs (Vinck 2009). Ces modes de conservation émergent plus pour répondre à un problème sociotechnique singulier (résoudre le décalage des calendriers de naissance et de consommation, s'adapter à des modes alimentaires qui privilégient les morceaux de découpe) que dans une perspective réduite parfois à tort à de la communication marketing agroalimentaire. Il s'agit de dispositifs marchands permettant d'organiser les liens que les produits entretiennent avec les sociétés (Callon 2016). La conservation dans des emballages dédiés remplit ainsi d'autres fonctions que celles qui lui sont données au premier abord, en assurant l'accès à de nouveaux marchés. Cela permet aux populations touristiques estivales de consommer des viandes de lait, de reconsidérer l'organisation sociétale du repas, garantir la sécurité alimentaire des consommateurs, ou encore faciliter la préparation culinaire, tout en s'adaptant aux pratiques d'élevage parfois contraignantes. Les techniques de conservation se révèlent ainsi être des opérateurs de désancrage/réancrage du produit vers un espace social marchand (Granovetter 1985). En cela, conserver relève d'une capacité à pouvoir et à savoir vendre, et permet aussi de faire revivre des produits menacés.

\section{Atelier de découpe individuel en Sardaigne}

Dans cette ferme familiale, l'agrément sanitaire HACCP est donné à l'exploitation afin de pouvoir abattre, découper et commercialiser les agneaux vers la structure de restauration. 


\section{Notes}

1. Le terme nustrale (« le nôtre») désigne en Corse une référence à l'origine insulaire, par différenciation de ce qui vient d'ailleurs. Le porc nustrale se différencie, par exemple, des charcuteries issues de carcasses importées et fait dans ce cas précis référence à la «race nustrale» ou «porcu neru » (porc noir). Dans le cas des agneaux, le terme est régulièrement employé soit pour se référer à une alimentation au lait maternel, soit aux pratiques de consommation à Noël et à Pâques.

2. Dispositif national de reconnaissance de la qualité des produits agricoles mis en œuvre par l'Institut national des appellations d'origines (INAO). Le régime des indications géographiques concerne plus précisément les caractéristiques d'un produit associées à un lieu spécifique de production (ex: la clémentine de Corse).

3. Plus de $80 \%$ des cabris sont actuellement abattus à la ferme alors que pour les éleveurs ovins, cette forme de commercialisation est résiduelle, dans la mesure où ils livrent leurs agneauxaux coopératives

4. Les abattoirs de Cuttoli et de Ponte Leccia sont des structures d'abattage multi-espèces traitant principalement des porcs et des bovins.
5. Dans le jargon usuel, le terme groupement de Roquefort est habituellement employé pour désigner le groupement des apporteurs affiliés à la société fromagère corse implantée à Lucciana. La référence à Roquefort perdure dans la mesure où ce groupement a une filiation directe avec le bassin de production de l'Aveyron. L'entreprise fromagère a collecté du lait en Corse durant tout le xxe siècle jusqu'à l'obtention de l'appellation où son rayon de collecte s'est alors re-territorialisé en Aveyron.

6. Dans le calendrier agro-pastoral qui a perduré jusqu'à la fin du xixe siècle, la période naturelle de mises bas des brebis en Corse se situait en fin d'hiver, notamment dans le cadre de la double transhumance où la lactation était prolongée jusqu'à la fin de l'été et les agneaux étaient vendus à Pâques avant la montée des animaux vers les estives.

7. Hazard Analysis Critical Control Point: méthode de maîtrise de la sécurité alimentaire des produits introduite dans l'Union Européenne en 1993. Ce référentiel permet à une entreprise de se doter de son propre système de contrôle avec l'appui d'un organisme certificateur qui lui donne une accréditation spécifique au-delà du régime réglementaire.

\section{I'auteur}

Nicolas Lacombe conduit des activités de recherche en Corse et en Sardaigne où il travaille sur la valorisation des productions agricoles alimentaires et non alimentaires. Il s'intéresse notamment à la place qu'occupent les coproduits dans ces territoires et a engagé récemment des recherches sur les laines ainsi que les cornes associées à l'artisanat, trajectoire de recherche qu'il inscrit dans le champ de l'économie circulaire.

\section{Iconographie}

Image d'ouverture. Carcasses entières de cabris vendues à la ferme (Lacombe, Corse). La présentation des carcasses en vente directe est codifiée par une série de critères, dont la mise en place de la crépine sur la cage

\section{Références}

Akrich, M. 1993 «Les formes de la médiation technique», Réseaux 60: 87-98.

Anzalone, G. 2005 «La viande comme marchandise (enquête)», Terrains \& travaux 2 (9): 125-142. thoracique qui permet d'éviter un dessèchement des parties intérieures. (C) N. Lacombe.

$1 \& 2$. $(9$ ODARC, Corse.

3, 4, $5 \& 6$. (C) N. Lacombe.
Bérard, L. \& P. Marchenay 2000 «Le vivant, le culturel et le marchand: les produits de terroir », Autrement. Série mutations 194: 191-216. 
Callon, M. 2013 «Qu'est-ce qu'un agencement marchand? » in $\mathrm{M}$. Callon et al. Sociologie des agencements marchands. Paris: Presses des Mines: 325-440.

- 2016 « Revisiting marketization: From interface-markets to market-agencements », Consumption Markets \& Culture 19: 17-37.

Callon, M., Méadel, C. \&V. Rabeharisoa 2000 «L'économie des qualités», Politix 13(52) $4^{e}$ trimestre: 211-239.

Cochoy, F. 2002 Une sociologie du packaging ou l'âne de Buridan face au marché. Les emballages et le choix du consommateur. Paris: P.U.F.

Cochoy, F. \& S. Dubuisson-Quellier 2000 «Introduction. Les professionnels du marché: vers une sociologie du travail marchand », Sociologie du travail, vol. XLII : 359-368.

Delfosse, C. 2010 «L'héritage en action: les relances et les territoires » in A. Berger, P. Chevallier, M. Dedeire \& G. Cortes Héritages et trajectoires rurales. Paris: l'Harmattan: 45-66.

Delfosse, C. \& J.-A. Prost 1998 «Transmission et appropriation des savoirs fromagers: un siècle de relations entre industriels de Roquefort et transformateurs corses», Ruralia 2 [En ligne] : ruralia.revues.org/27.

Delfosse, C. \& C. Bernard 2007 «Vente directe et terroir», Méditerranée 109 [En ligne] : mediterranee.revues. org/108.

Diaz-Bone, R. \& L. Thévenot 2010 «La sociologie des conventions, élément central des nouvelles sciences sociales françaises», Trivium 5 [En ligne] : journals. openedition.org/trivium/3626.

Escala, T. 2006 «La production d'une figure du producteur. Le cas d'un label rouge», Économie rurale 293: 38-54.
Granovetter, M. 1985 «Economic action and social structure: The problem of embeddedness », American Journal of Sociology 91 (3): 481-510.

Karpik, L. 1989 «L'économie de la qualité», Revue française de sociologie 30 (2) : 187-210. DOI : 10.2307/3321761.

— 2007 L'économie des singularités. Paris: Gallimard.

Lacombe, N. 2015 Les coproduits entre marginalisation et relance. Le cas des viandes de petits ruminants en élevage méditerranéen. Thèse de doctorat en Géographie. Corte: Université de Corse Pascal Paoli.

Lacombe, N. et al. 2012 «Labattage fermier en Corse: tensions ente normes réglementaires et légitimation d'une pratique socialement acceptée» in $19^{e}$ Rencontres Recherches Ruminants. Paris: INRA / Idele. [En ligne] : journees3r.fr/spip.php?article3428.

Lambert, J.-L. 1986 Lévolution des modèles de consommation alimentaire en France. Thèse de doctorat. Nantes.

Poulain, J.-P. 2002 Sociologies de l'alimentation. Paris: PUF.

Senil, N. et al. 2014 «Le patrimoine au secours des agricultures familiales? Éclairages méditerranéens », Revue Tiers-Monde 220 (4): 137-158.

Stanziani, A. 2003 La qualité des produits en France, XVIII $x x^{e}$ siècles. Paris: Belin.

Sylvander, B. 1995 «Conventions de qualité, marchés et institutions: le cas des produits de qualité spécifique» in F. Nicolas \& E. Valceschini dir. Agroalimentaire. Une économie de la qualité: 167-183.

Vinck, D. 2009 « De l'objet intermédiaire à l'objet-frontière », Revue d'anthropologie des connaissances 3 (1): 51-72.

\section{Pour citer l'article}

Lacombe, N. 2018 «Inventer le marché par la conservation. Le cas des viandes de lait en Corse et en Sardaigne», Techniques\&Culture 69 «Le temps des aliments», p. 136-147. 